\title{
Handlungsempfehlung KLR und CAFRAD
}

Die Umsetzung der neuen Weiterbildungsordnung führt dazu, dass Radiologen*innen gebeten werden, für Kollegen* innen Sachkunden für die Erlangung von Fachkunden im Strahlenschutz zu bestätigen. Die KLR, CAFRAD und die DRG haben daher gemeinsame Handlungsempfehlungen für verantwortliche Radiologen*innen entwickelt, die die bestehenden Regeln zusammenfasst, ihre Bedeutung bewertet und die Einhaltung der Regeln empfiehlt.

\section{Hintergrund}

Die neue Weiterbildungsordnung (WBO) fordert für den Erwerb diverser Facharztund Schwerpunktkompetenzen fachgebietsspezifische Fachkunden im Strahlenschutz. Die Voraussetzungen hierfür werden unter „Handlungskompetenz“ bzw. „diagnostische Verfahren“ fachspezifisch definiert und orientieren sich inhaltlich an den entsprechenden Richtlinien zum Erwerb der Sach- und Fachkunde (Richtlinie Fachkunde und Kenntnisse im Strahlenschutz bei dem Betrieb von Röntgeneinrichtungen in der Medizin oder Zahnmedizin vom 22. Dezember 2005, geändert durch Rundschreiben vom 27.06.2012, BMU - RS II 4-11603/01). Details hierzu in der synoptischen Übersicht am Ende des Dokumentes.

Da in der WBO für die einzelnen Facharztund Schwerpunktbezeichnungen der Begriff „erforderliche Fachkunde“ nicht definiert wird, könnten hierfür grundsätzlich die Fachkunden Rö2 (Notfalldiagnostik), Rö3.1 (Skelett), Rö3.2 (Thorax), Rö3.3 (Abdomen), Rö 3.4 (Mamma), Rö 3.5 (Gefäße ohne Herz), Rö4 (sonstiges begrenztes Anwendungsgebiet), Rö6 (Kinder), Rö7 (Interventionen) sowie Rö9.1 oder Rö9.2 (DVT) gefordert werden. Verschiedene Landesärztekammern haben inzwischen die Formulierung „erforderliche Fachkunde“ für die einzelnen Gebiete näher definiert, wobei unklar bleibt, auf welcher Basis diese Definition erfolgte. Die Verknüpfung von Weiterbildungsordnung und Strahlenschutzrecht (Fachkunden) ist aus Sicht der Radiologie problematisch, da das Ziel der Fachkunden die Vermittlung des erforderlichen Wissens zur sicheren Anwendung von Röntgenstrahlung unabhängig von der Berufszugehörigkeit ist.

\section{Problemstellung}

Im Ergebnis ist in der neuen WBO der jeweils geforderte Sachkundenachweis Voraussetzung, um zur Prüfung in der entsprechenden Facharzt- und Schwerpunktkompetenz zugelassen zu werden. Ferner ist es für den Weiterbildungsbefugten notwendig, die Voraussetzungen für die Erlangung dieser Fachkunde auch der Ärztekammer bei der Beantragung der Weiterbildungsbefugnis nachzuweisen. Gelingt ihm dies nicht, besteht das Risiko, wegen Fehlen dieser Voraussetzung nicht die Befugnis für die volle Weiterbildungszeit zu erhalten.

- Basierend auf dieser Situation ist zu erwarten, dass Radiologen*innen verstärkt gebeten werden, für Kollegen*innen anderer Fächer die in der neuen WBO geforderte Sachkunde zur Erlangung der Fachkunde zu bestätigen. In vielen Fällen liegen die Voraussetzungen, insbesondere die Zeiten und Untersuchungsfrequenzen unter Anleitung des fachkundigen Arztes für den Sachkundeerwerb und damit den Fachkundeerwerb jedoch nicht vor.

\section{Risiken der Verknüpfung von Weiterbildungsordnung und Strahlenschutzrecht}

Die Notwendigkeit der Bescheinigung der Sachkunde im Rahmen der neuen WBO geht mit vielfältigen Risiken einher:

- Die in den meisten Fällen erforderliche Rotation in die Radiologie ist im klinischen Alltag für die anderen Fächer schwer abbildbar. In Zeiten der Personalverknappung sinkt die erforderliche Bereitschaft in einzelnen Fächern zur „Freistellung“ von WB-Assisten*innen. Mit Verweis auf umfangreiche radiologische Fallkonferenzen oder intraoperatives Röntgen im jeweiligen Fachbereich wird oftmals bereits das Vorliegen einer ausreichenden Sachkunde bei den Vertretern der anderen Fächer angenommen und damit wesentliche
Voraussetzungen des Sachkunde- und Fachkundeerwerbes missachtet. Fallkonferenzen ersetzen die Zahl der unter Aufsicht zu befundenden Untersuchungen nicht. Intraoperatives Röntgen kann nur für eine Sachkunde Rö4 genutzt werden und auch nur dann, wenn die Untersuchungen unter Aufsicht des fachkundigen Arztes erfolgten, der die Bescheinigung letztendlich ausstellt.

- Fehlende Transparenz und Konzeption begünstigen interdisziplinäre Konfliktsituationen. In einem anzunehmenden Graubereich ist zu erwarten, dass versucht wird, auf die Radiologie über entsprechenden Druck innerhalb medizinischer Einrichtungen zur Bescheinigung zu drängen ohne dass weder die Anzahl von Befundungen nachweisbar durchgeführt wurde und dokumentiert ist, noch die erforderlichen Zeiten abgeleistet wurden.

- In einzelnen Fällen wird die notwendige Sachkunde von den einzelnen Fächern über entsprechende Weiterbildungsbefugte mit der erforderlichen Fachkunde selbst bescheinigt. Das ist prinzipiell möglich, wenn in der jeweiligen Abteilung ein Arzt mit der entsprechenden Fachkunde tätig ist. Das gilt jedoch nur dann, wenn auch die Infrastruktur hierfür gegeben ist: Ohne eigenverantwortlich betriebenes Röntgengerät sowie eigenes Assistenzpersonal und die entsprechenden Untersuchungszahlen kann auch ein fachkundiger Orthopäde oder Unfallchirurg das Sachkundezeugnis nicht ausstellen.

- Bei der Umsetzung der neuen Weiterbildungsordnung können die einzelnen Ärztekammern den Umfang der erforderlichen Fachkunde in ihrem Geltungsbereich individuell festlegen. Das kann zu Problemen beim Wechsel der Kammergebiete führen - bis hin zur Verweigerung der entsprechenden Weiterbildungsermächtigung.

\section{Handlungsempfehlungen}

Sachkundebescheinigungen durch Radiologen*innen werden nur nach entsprechender Rotation in die Radiologie und un- 
ter strenger Einhaltung der vorgegebenen Kriterien der Fachkunderichtlinie vergeben. Wesentlicher Bestandteil der Standardisierung und Grundlage der Sachkundebescheinigung ist die verpflichtende und damit rechtssichere Dokumentation der durchgeführten Befundungen in der radiologischen elektronischen Dokumentation entsprechend den geltenden strahlenschutzrechtlichen Vorgaben.

Als Hilfestellung für die Umsetzung werden folgende Handlungsempfehlungen gegeben:

- Sachkundebescheinigungen werden nur nach entsprechender Rotation in die Radiologie und unter strenger Einhaltung der vorgegebenen Kriterien der Fachkunderichtlinie vergeben.

- Der Sachkundeerwerb außerhalb der Radiologie durch entsprechend Weiterbildungsbefugte mit der erforderlichen Fachkunde setzt eine entsprechende Infrastruktur voraus. Ohne Röntgengerät, eigenes Assistenzpersonal und die entsprechenden Untersuchungszahlen kann auch ein fachkundiger Arzt außerhalb der Radiologie das Sachkundezeugnis nicht ausstellen.

- Im Falle einer interdisziplinären Konfliktsituation sollten Gespräche protokolliert und mit den Hinweisen versehen werden, dass das Geforderte einen Rechtsverstoß darstelle und daher nicht mitgetragen werden könne. Gleichzeitig sollte über das Angebot einer Rotation die aktive Mitarbeit und damit eine Lösung des Problems vermerkt werden.

- Die Rotation der Kolleg*innen in die Radiologie kann in Vollzeit oder Teilzeit (arbeitstäglich) erfolgen. „Arbeitstäglich“ erlaubt auch die stundenweise Rotation. Daher kann der Zeitraum der Rotation tages- wie stundenweise den lokalen realen Bedingungen angepasst werden. Dies erhöht Flexibilität und Akzeptanz von Rotationsmodellen innerhalb des Kollegiums. Prinzipiell sollte dabei eine Form der Rotation Vorrang eingeräumt werden, die einen Wissenstransfer in beide Richtungen begünstigt

- Beachtung der Möglichkeiten zur Reduzierung der Mindestzeiten. Doppelte Verkürzungen sind nicht zulässig: beispielsweise 50 \% Sachkundeerwerb in einer hauptamtlichen Radiologie mit Weiterbildungsermächtigung und dann nochmals $50 \%$ bei Erwerb der Sachkunde in einen zweiten Organsystem

- Erfassen der durchgeführten Befundung mit OA/FA Validierung in einer elektronischen radiologischen Dokumentation, die den Anforderungen der geltenden strahlenschutzrechtlichen Vorgaben genügt - RIS oder ähnliche Systeme.

- Nur die im Rahmen der Rotation selbständig durchgeführten Befundungen werden für die Zahlen angerechnet. Eine pro-Forma Integration in die Befunde ohne tatsächlich Beteiligung an der Befundung ist nicht zulässig.

- Die Zahl der Befunde sowie die erforderliche Zeit zum Sachkundeerwerb kann nicht über klinische Fallkonferenzen oder Tumorboards reduziert werden.

- Intraoperative „radiologische Befundkontrollen “ können nur im Rahmen der Fachkunde Rö4 (intraoperatives Röntgen) und dann nur durch den direkt Aufsicht führenden fachkundigen Arzt angerechnet werden

- Die mögliche Überprüfbarkeit der ausgestellten Sachkunde aufgrund der radiologischen elektronischen Dokumentation sind sehr handfeste Argumente für dieses Vorgehen.

- Für Praxen, MVZ, Institutionen ohne hauptamtliche Radiologie muss die genaue Ausgestaltung der Rotation individuell abgesprochen werden, da in diesen Settings die Realisation durch die andere Arbeitsorganisation deutlich anspruchsvoller ist und die Betreuung durch Fachärzt* innen effektiv Zeit benötigt. Wenn auch aus organisatorischen Gründen keine Rotation möglich ist, sollte auf entsprechende hauptamtliche Weiterbildungsbetriebe der Radiologie verwiesen werden.

\section{Anhang}

Für eine ausführliche Erläuterung der Richtlinie für Fachkunde und Sachkunde verweisen wir auf die Publikation von Herrn Loose aus dem Jahr 2017:
[Loose R, Wucherer M, Fachkunderichtlinien und Kurse zur Qualifikation. Der Radiologe. 2017 Jul; 57(7): 529-533. doi:10.1007/s00 117-017-0249-4].

\section{Synoptische Übersicht „Richtlinie Fachkunde und Kenntnisse im Strahlenschutz"}

Festgelegt in den Regeln für den Sachkunde- und Fachkundeerwerb sind eine Zeit zum Sachkundeerwerb von 3-12 Monaten und eine bestimmte Anzahl von unter fachkundiger Aufsicht durchgeführten und befundeten Röntgenuntersuchungen (Details siehe Richtlinie BMU-RS II4-11603/01). Die Richtlinie unterscheidet zwischen Sachund Fachkunde.

Sachkunde = praktische Erfahrung $=$ Anzahl von durchgeführten/ befundeten Untersuchungen und Zeitraum in dem diese durchgeführt werden. Dies beinhaltet auch die Stellung der rechtfertigenden Indikation.

Fachkunde = Befähigung zur eigenverantwortlichen Durchführung (=Indikation +technische Durchführung+Befundung) von Röntgenuntersuchungen = Kurse (mindestens Kenntnisse, Grund- und Spezialkurs) + Sachkunde

In Hinblick auf die erforderliche Sachkunde gibt die Richtlinie Vorgaben als auch Hinweise für Handlungsspielraum:

\section{Inhaltlicher Umfang}

- Die Sachkunde wird unter Anleitung, ständiger Aufsicht und Verantwortung eines Arztes, der auf dem betreffenden Anwendungsgebiet die erforderliche Fachkunde im Strahlenschutz besitzt (kann also auch ein Nichtradiologe* in sein), und durch den Nachweis einer ausreichenden Anzahl dokumentierter Untersuchungen und Zeiten erworben.

- Zur Erreichung der geforderten Anzahl dokumentierter Untersuchungen sind die drei Elemente a) rechtfertigende Indiktion, b) technische Durchführung und c) Befundung in angemessener Gewichtung zu berücksichtigen. 
- Nicht erforderlich ist, dass der die Sachkunde Erwerbende eine Mindestzahl von Untersuchungen auch selbst in vollem Umfang technisch durchführt; insbesondere dürfen keine ungerechtfertigten Röntgenuntersuchungen technisch durchgeführt werden, um das Ausbildungsziel zu erreichen. Allerdings muss sichergestellt sein, dass die technische Durchführung in angemessenem Umfang praktisch erlernt wird.

- Die Sachkunde im Strahlenschutz, z. B. das Stellen des richtigen Befundes an Hand der Röntgenbilder, darf erforderlichenfalls zum Teil auf der Grundlage einer Fallsammlung erworben werden.

- Für die Fachkunde Rö4 „intraoperatives Rönten “ können zusätzlich intraoperative „radiologische Befundkontrollen“ angerechnet werden sofern sie durch einen fachkundigen Arzt beaufsichtigt werden.

\section{Zeitlicher Umfang}

- Der Sachkundeerwerb erfordert im Regelfall eine arbeitstägliche (nicht gleichzusetzen mit ganztägige) Rotation entsprechender WBAssistent*innen in die Radiologie.

- Die Voraussetzungen für eine Reduktion der Mindestzeiten (z. B. von 12 auf 6 Monate) werden in der Richtlinie dezidiert genannt.

a) Reduktion jeweils auf die Hälfte, wenn die Sachkunde ganztägig in einer fachradiologischen Abteilung mit Weiterbildungsbefugnis und dem erforderlichen Leistungsumfang erworben wird.

b) Reduzierung der Mindestzeiten bei Erwerb der Sachkunde in mehr als einem Organsystem möglich, wenn bereits die Fachkunde für ein Anwendungsgebiet erfolgreich erworben wurde und die gegebenenfalls erforderliche Aktualisierung nachgewiesen ist. In diesem Fall verkürzt sich die
Mindestzeit für jedes weitere Anwendungsgebiet um die Hälfte.

Die Anzahl der dokumentierten Untersuchungen verringert sich entsprechend.

- Die zu dokumentierenden Untersuchungen müssen nicht zusammenhängend erbracht werden.

\section{Dokumentationspflichten}

- Die Anwendungszahlen und Mindestzeiten sind in einem Tätigkeitsbericht aufzuzeichnen und von einem aufsichtsführenden fachkundigen Arzt monatlich zu bestätigen.

- Der Erwerb der Sachkunde ist durch Zeugnisse nachzuweisen.

J. Wessling (CAFRAD); G. Antoch (DRG), R. Loose (DRG), J. Lotz (KLR), 